Annales Geophysicae (2003) 21: 819-832 (C) European Geosciences Union 2003

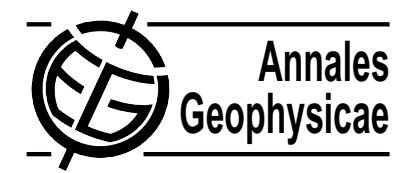

\title{
Detecting quasi-oscillations in the monthly precipitation regimes of the Iberian Peninsula
}

\author{
L. Morala, A. Serrano, and J. A. García \\ Departamento de Física, Facultad de Ciencias, Universidad de Extremadura, Badajoz, Spain
}

Received: 13 May 2002 - Revised: 26 August 2002 - Accepted: 28 August 2002

\begin{abstract}
A spectral analysis of the time series corresponding to the main monthly precipitation regimes of the Iberian Peninsula was performed using two methods, the MultiTaper Method and Monte Carlo Singular Spectrum Analysis. The Multi-Taper Method gave a preliminary view of the presence of signals in some of the time series. Monte Carlo Singular Spectrum Analysis discriminated between potential oscillations and noise.
\end{abstract}

From the results of the two methods it is concluded that there exist three significant quasi-oscillations at the $95 \%$ level of confidence: a 5.0 year quasi-oscillation and a longterm trend in the Atlantic pattern of March, a 3.2 year quasioscillation in the Cantabrian pattern of January, and a 4.0 year quasi-oscillation in the Catalonian pattern of February. These quasi-oscillations might be related to climatic variations with similar periodicities over the North Atlantic Ocean.

The possible simultaneity of high values of precipitation generated by the significant quasi-oscillations and high sealevel pressures was studied by means of composite maps. It was found that high values of precipitation generated by the oscillations of the Atlantic patterns of January and March exist simultaneously with a specific high pressure structure over the North Atlantic Ocean, that allow cyclonic perturbations to cross the Iberian Peninsula. During the non-wet years, this high pressure structure moves northwards, keeping the track of the low pressure centers to the north, far from the Iberian Peninsula.

On the other hand, high values of precipitation generated by the oscillation of the Cantabrian pattern of January exist simultaneously with a high pressure structure over the Galicia region and the Cantabrian Sea, that allow a northerly flow over the region.

Also, a positive trend in the NAO index for March has been found, starting in the sixties, which is not evident for other winter months. This trend agrees with the decreasing trend found in the March Atlantic pattern.

Correspondence to: J. A. García (agustin@unex.es)
Key words. Meteorology and atmospheric dynamics (climatology; precipitation) Oceanography: general (climate and interannual variability)

\section{Introduction}

Precipitation is a very irregular variable. It presents a wide variability both spatially and temporally at very different scales (interannual and intraannual). This is particularly true for the Iberian Peninsula, whose geographical location (between two sources of humidity: the Atlantic Ocean and the Mediterranean Sea) and the existence of various mountain chains add difficulties to the construction of a precipitation model. However, the great influence of precipitation on life in the Iberian Peninsula (agriculture, water supply, the tourist trade, etc.) makes it of great importance to understand the causes of this variability. In this sense, the detection of oscillations in precipitation time series is a very interesting topic, not only for predictive purposes, but also because it yields important information for the understanding of climate, since the oscillations can be seen as responses of the climate system to external forcing or feedback processes.

Precipitation over the Iberian Peninsula shows a strong seasonal character which affects its nature (frontal or convective). This is due to the fact that some factors become important only during some months of the year. Thus, while precipitation during winter can be mostly explained by synopticscale perturbations crossing the Iberian Peninsula, local factors generating convective storms must be taken into account for the understanding of Spring, Summer and Autumn precipitation. This situation suggests considering separately each calendar month in order to improve the characterization of the precipitation regimes in the Iberian Peninsula. This was done by Serrano et al. (1999a), who showed that some monthly precipitation regimes exist only during certain months of the year, and vanish for others.

In the present paper a spectral analysis of those significant monthly precipitation regimes is performed. The aim is 
Table 1. Rank and percentage of total variance explained (in parentheses) by PCs that are associated with common patterns. Blank spaces correspond to months where the pattern was not detected

\begin{tabular}{lccccccc}
\hline Month & ATL & INT & SUR & POR & CAT & LEV & CAN \\
\hline JAN & $1(33.5)$ & & $2(18.1)$ & & $3(11.9)$ & $5(8.0)$ & $4(8.7)$ \\
FEB & $1(42.8)$ & & $2(10.0)$ & & $5(6.9)$ & $3(9.2)$ & $4(8.0)$ \\
MAR & $1(36.5)$ & & $4(8.4)$ & & $2(11.9)$ & $3(9.6)$ & $5(8.3)$ \\
APR & & $2(16.3)$ & $1(17.0)$ & $5(9.8)$ & $4(11.5)$ & $3(11.8)$ & $6(8.7)$ \\
MAY & & $2(12.4)$ & $1(19.3)$ & $6(5.9)$ & $4(10.4)$ & $3(10.8)$ & $5(9.8)$ \\
JUN & & & $1(17.3)$ & $5(7.2)$ & & $2(11.7)$ & $3(11.4)$ \\
JUL & & & & $4(9.5)$ & & & $5(8.7)$ \\
AUG & & $2(12.6)$ & $5(8.7)$ & $7(5.7)$ & $4(11.7)$ & $3(12.4)$ & $6(7.7)$ \\
SEP & & $3(10.7)$ & $1(21.6)$ & $6(8.3)$ & $7(8.1)$ & $4(10.0)$ & $5(9.7)$ \\
OCT & & $2(11.2)$ & & $3(10.4)$ & $5(8.4)$ & $4(8.9)$ \\
NOV & $1(28.3)$ & & $2(20.9)$ & & $3(12.2)$ & $5(7.9)$ & $4(9.1)$ \\
DEC & $1(26.2)$ & & & & & & \\
\hline
\end{tabular}

the detection of significant quasi-oscillations in the monthly precipitation series over the Iberian Peninsula by analyzing separately each calendar month. This study allows for the identification of time characteristics specific to some months that might be masked in annual or seasonal analyses.

There have been studies aimed at detecting oscillations in the main precipitation regimes in the Iberian Peninsula. However, they deal with annual series (Rodríguez-Puebla et al., 1998) or with continuous quarterly series (García et al., 2002). Since some spatial regimes of precipitation are only reliable for certain calendar months, an analysis for each month, as is performed in the present paper, seems to be more suitable.

The time analysis was carried out using two modern techniques of time series spectral analysis: MTM (the MultiTaper Method) and SSA (Singular Spectrum Analysis). The great number of data series to be analyzed suggested first making a preliminary exploration of the spectra. For this purpose, we used the MTM, which generates a first selection of quasi-cycles that later will be studied in detail using an SSA, in particular the Monte Carlo SSA test (Allen and Smith, 1996). Finally, we will describe the results of a search for a relationship between the resulting statistically significant quasi-cycles and some climatic variable, in order to explain the origin of the oscillations.

We will begin by describing briefly the data and the spectral analysis methods employed in this work (MTM and SSA).

\section{Data}

Precipitation is a very complicated variable to deal with. In the case of the Iberian Peninsula, for instance, its description involves a large range of temporal and spatial scales. The highly seasonal nature of its precipitation field suggests studying each calendar month separately, since no precipitation seasons could be defined a priori for all the stations. In order to study the evolution of the precipitation field and its possible teleconnections, the main modes of variation of precipitation over the Iberian Peninsula identified and characterized by Serrano et al. (1999a) were used in this work. These modes of variation are preferred over the individual station records, since they better represent the general main precipitation regimes and are less noisy than the local records.

The main modes of variation of precipitation, which are the basis of this study, were obtained by applying Principal Component Analysis (PCA) to forty precipitation station records covering the Iberian Peninsula from 1919 to 1992 (Serrano et al., 1999a). Thirty-five precipitation series were provided by the Instituto Nacional de Meteorologia of Spain, four by the Instituto Nacional de Meteorologia e Geofisica of Portugal and one by the Real Instituto y Observatorio de la Armada Española of Spain. The modes of variation were obtained for each calendar month separately. The PCs were rotated using the Varimax method. Direct Oblimin and Promax were also tested. Since there were no significant differences between the rotated PCs, the simpler and widely used Varimax method was preferred.

Under the hypothesis that the PCs are linked to general circulation conditions that vary slowly throughout the year, main patterns must remain relevant for various months. Accordingly, only those spatial patterns appearing during at least two contiguous calendar months were retained. Thus, taking into account the different months, 57 modes of variation were selected. These modes of variation correspond to seven different precipitation patterns: ATL (Atlantic), INT (Interior), SUR (South), POR (Galicia and Northern Portugal), CAT (Catalonia), LEV (Levante) and CAN (Cantabrian).

The rank in the PCA, the percentage of total variance explained and the pattern they are associated with of the 57 modes of variation are listed in Table 1 . Note that not all patterns are detected in each calendar month. It can be seen that during summer only POR and CAN patterns are found. This is due to the increasing importance of local factors which 


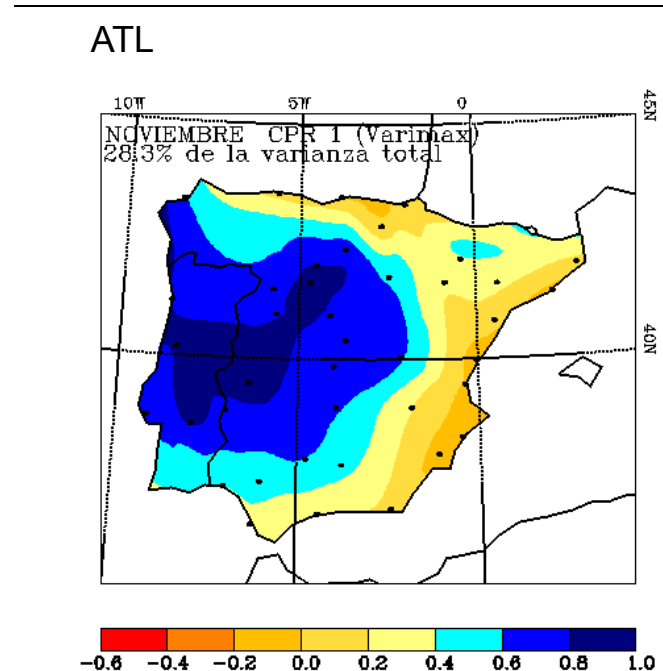

INT
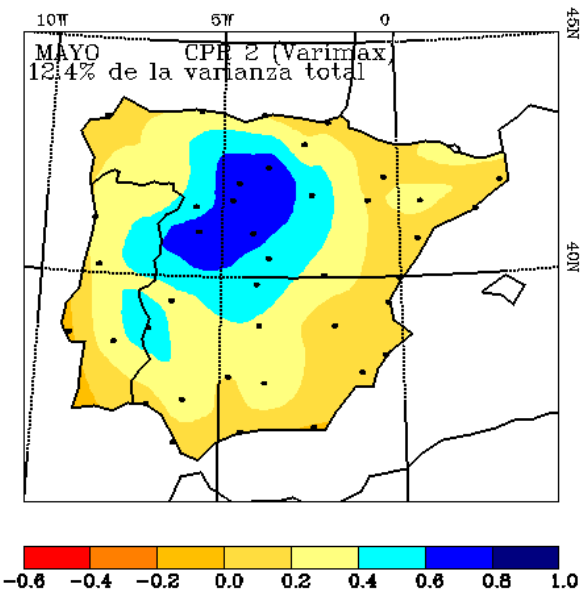

\section{SUR}

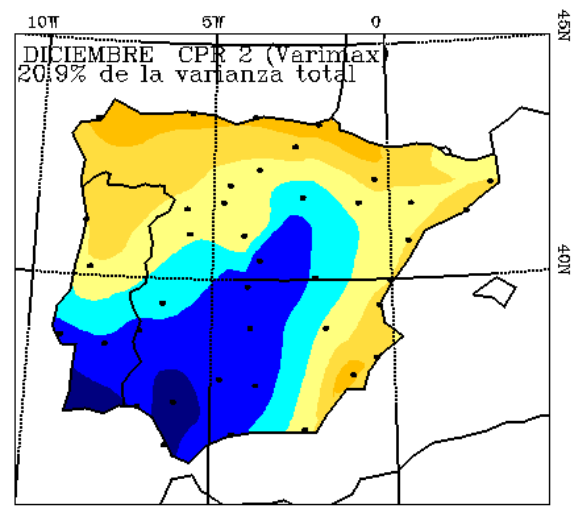

POR
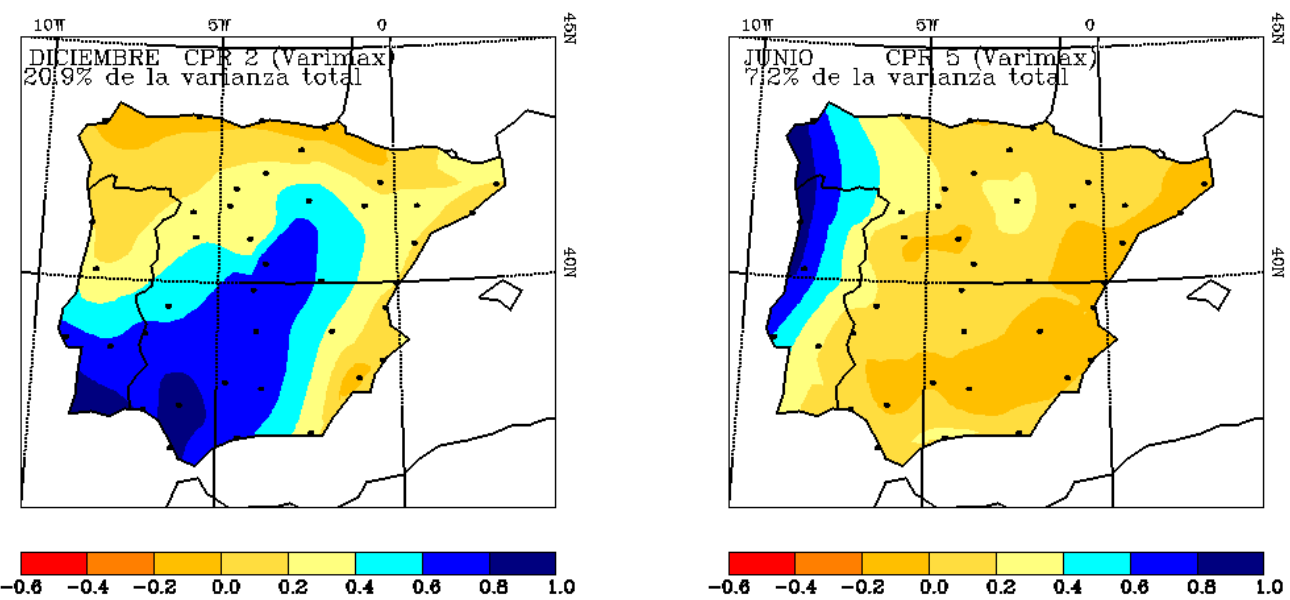

Fig. 1. Examples of loading maps of the ATL, INT, SUR, POR, CAT, LEV, and CAN patterns. The text gives the month where the pattern appears and the percentage of variance explained by the pattern for that month.

block the detection of wide, spatially coherent patterns of precipitation. In Fig. 1 an example of the spatial distribution loading for each pattern is shown. The loading value represents the correlation between the station series and the PC, since Hotelling normalization was used (Jollife, 1986).

These 57 modes of variation associated with seven different precipitation patterns constitute a suitable description of the monthly precipitation field over the Iberian Peninsula and are the basis of the present study. For a more detailed description of these modes of variation, please refer to Serrano et al. (1999a).

\section{Spectral analysis methods}

While a time series can be analyzed in either the time-domain or the frequency-domain, the latter is usually more interesting, because the relevant temporal scales of the different physical processes are easier to distinguish. In order to obtain a good estimate of the distribution of the power (variance) of the series versus frequency (the power spectral density), it is reasonable to apply independent methods. We selected the following:

1. The Multi-Taper Method (MTM);

2. Singular Spectrum Analysis (SSA): the Monte Carlo SSA test (MCSSA).

\subsection{Multi-Taper Method (MTM)}

The purpose of this non-parametric spectral method (Thomson, 1982) is to compute a set of independent and significant estimates of the power spectrum, in order to obtain a better and more reliable estimate for finite time series than with single-taper methods. The MTM provides high spectral resolution, as well as statistical confidence levels for the 


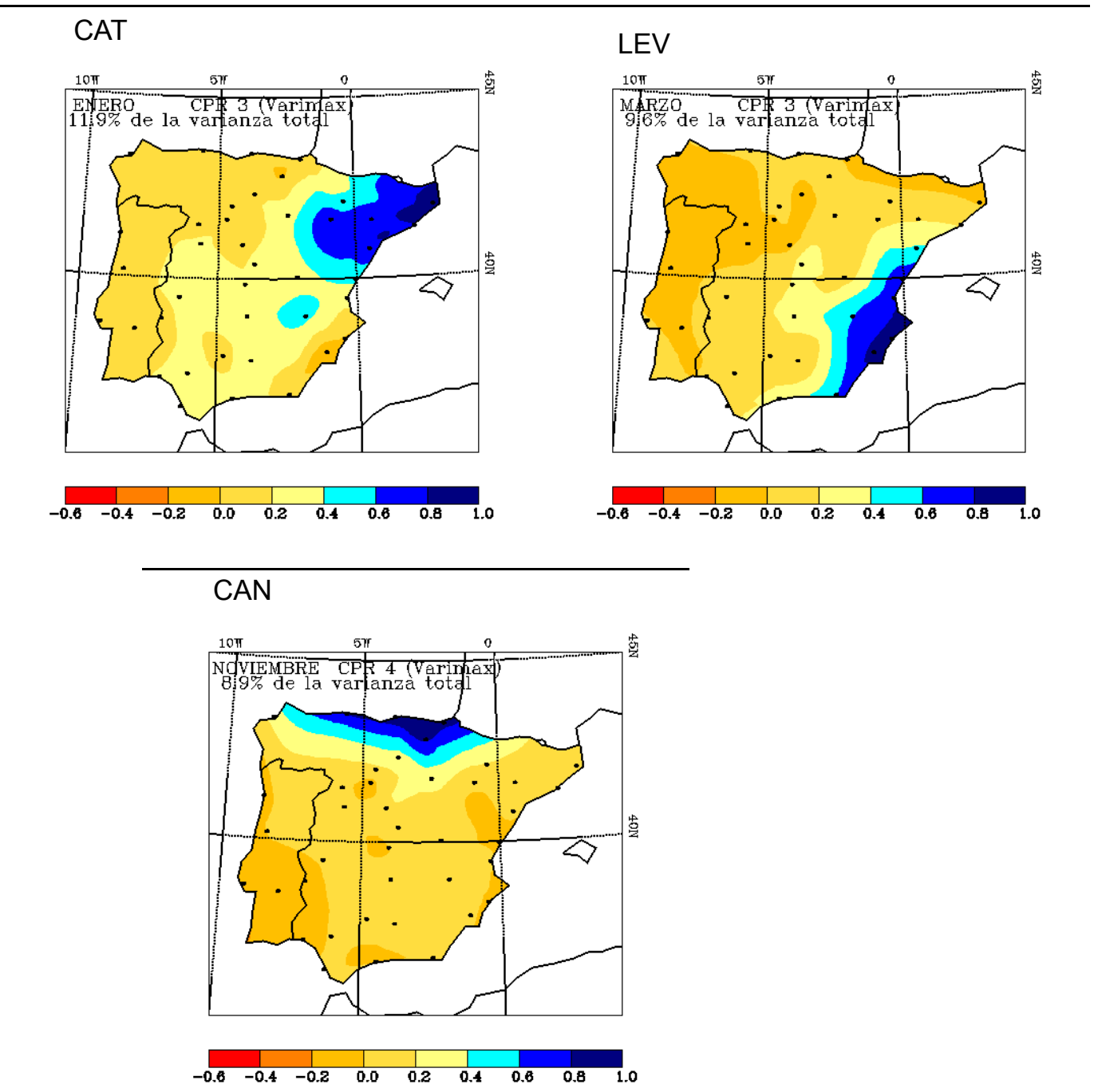

Fig. 1. Continued ....

spectral peaks it detects. It is thus superior to the classical Blackman-Tukey method, which yields a much lower resolution, and because the confidence levels are independent of the peak amplitudes. The MTM has been applied to various fields: Earth sciences, (Lindberg, 1986; Park et al., 1987), geophysics (Lanzerotti et al., 1986), climatology on interdecadal and century time scales (Kuo et al., 1990; Ghil and Vautard, 1991; Mann et al., 1995) and paleoclimatology with tree-ring data (Thomson, 1990b), marine core data (Thomson, 1990a; Berger et al., 1991), and ice core data (Yiou et al., 1995).

The method, devised by Thomson (1982) based on the work of Slepian (1978), consists of objectively finding tapers in order to minimize the spectral leakage of the power spectrum outside a pre-determined bandwidth. Thomson also shows that only $K=2 p-1$ tapers are resistant to spectral leakage, where $p$ is the the half-bandwidth expressed in Rayleigh frequency units. Thus, only $K$ tapers are used in the calculations.
The method provides an unbiased estimate of the amplitude in the case of a white-noise background, and is robust to different types of noise and signal patterns. Once the MTM spectrum has been obtained it is necessary to isolate any periodic signal corresponding to singular peaks in the power spectrum. This is accomplished by Thomson's (1982) reshaping procedure with some slight modifications (Mann and Lees, 1996): the robust noise background estimation procedure. This procedure uses a median-smoothed MTM spectrum of the time series in order to provide an estimate of the underlying noise background. Assuming that this noise was generated by an $\mathrm{AR}(1)$ red noise process, the true noise background is obtained by fitting an analytical red noise spectrum to the median-smoothed background estimate. Finally, the significance of periodic or quasi-periodic peaks in the spectrum relative to the estimated red noise background is gauged by using elementary sampling theory (Percival and Walden, 1986). Elementary sampling theory, used with a single data taper by Gilman et al. (1963) in their investigation of red 
noise confidence testing in climate spectra, assumes that the spectra are $\chi^{2}$ distributed with $v$ degrees of freedom in the spectral estimate. For the adaptive multi-taper spectrum estimate $v \approx 2 K$. The ratio of power associated with a peak in the spectrum to the local power level of the background noise is assumed to be distributed as $\frac{\chi^{2}}{v}$, and can be compared to the tabulated $\chi^{2}$ probability distribution to determine peak significances.

\subsection{Singular Spectrum Analysis (SSA): a tool for studying} dynamical systems

Singular Spectrum Analysis (SSA) is a data-adaptive method based on the idea of sliding a window down a time series and looking for patterns that account for a high proportion of the variance of the series obtained. This analysis is closely related to the technique of principal component analysis. The original purpose of SSA was noise reduction in the analysis of experimental data and was first applied to nonlinear dynamics by Broomhead and King (1986). Paleoclimatic records were analyzed by SSA by Fraedrich (1986) and Fraedrich and Ziehmann-Schlumbohm (1994), who observed that the algorithm could be used to estimate the number of degrees of freedom necessary to model the dynamics of an attractor. Vautard and Ghil (1989) refined SSA and applied it to four long marine cores. They emphasized the direct physical interpretation of the individual EOFs (empirical orthogonal functions) obtained with SSA, introducing the idea of searching for pairs of sinusoidal EOFs in quadrature, which were taken to indicate a physical oscillation. Various other records have been analyzed through SSA (the historical global temperature record, the Southern Oscillation index) with the introduction of improvements in distinguishing signals from noise. One such problem had been the lack of effective statistical tests to discriminate between potential oscillations and noise. Allen and Smith (1996, henceforth AS96) found that the basic formalism of SSA provides a natural test for modulated oscillations against an arbitrary colored noise null hypothesis: the Monte Carlo SSA test (MCSSA).

\subsubsection{Applications of SSA}

1. Detecting signals. A natural application of SSA is detecting the signal in a time series. By plotting the eigenvalues in decreasing order, one can identify those EOFs dominated by a signal and those dominated by noise, discriminating between high variance oscillations and a steep slope, and noise characterized by low variances and a flat floor. The occurrence of a pair of high-ranked eigenvalues indicates the possible presence of a physically meaningful deterministic oscillation. While it is effective at separating signals from pure white noise, the rank-order is unreliable for systems contaminated with red noise or for nonlinear systems, in general. Highranked pairs could be spurious pairs. We need to decide on the confidence level to reject the null hypothesis that the features identified are attributable to the stochastic component of the record. There are two ways to test for statistical significance (Elsner and Tsonis, 1996): analytically or with the use of a Monte Carlo approach. Analytical methods involve assumptions about the distribution of the particular random variable being used as the test statistic. The distribution statistics on random variables from SSA will, in general, be non-Gaussian and therefore difficult to describe analytically. An acceptable way around this problem is to use the Monte Carlo approach. The Monte Carlo SSA test involves generating surrogate records from a model based on the null hypothesis, see AS96 for details. The application of the MCSSA test can be summarized as follows. The first step in MCSSA is to assume a noise model. Since a large class of geophysical processes generate series with large power at the lower frequencies (AS96), a red noise is a convenient model to begin with. From the time series $x(t)$, the parameters of the red noise are determined by a maximum-likelihood criterion. In order to test the statistical significance of a signal against the null hypothesis selected, an ensemble of surrogate time series is generated with the parameters obtained in the above step. At each Monte Carlo step an autocovariance matrix $C_{R}$ is computed. These covariance matrices are projected onto the EOFs of the actual data yielding an ensemble of eigenvalues from which the confidence intervals are obtained. Usually the 2.5 and 97.5 percentiles are computed. If an eigenvalue $\lambda_{k}$ lies outside this confidence interval, the time series can be considered to be different from the generic red noise simulation at the $95 \%$ level of significance.

As pointed out in AS96, the main problem with the above procedure is that data and surrogates are not treated in the same way, since both the data covariance matrix and the surrogate covariance matrices are projected onto the data EOFs. This compresses the variance into the highest-ranked EOFs in the data but not in the surrogates, since it is implicitly assumed that none of the data is noise. AS96 introduces a variant of the MCSSA method which is based on the assumption that all the data is noise, except that which has previously been established as signal. The procedure followed is then the same as before, except that data covariance matrix and surrogate covariance matrices are projected onto the null hypothesis basis, which is assumed initially to be red noise. If some of the data eigenvalues lie above the 97.5 percentile of their corresponding surrogate error bar, the data are taken to be inconsistent with the null hypothesis and those eigenvalues with their corresponding EOFs are taken as a signal. Once an eigenvalue and the corresponding EOF is taken as signal, a new Monte Carlo test is carried out including the EOFs found to be significant in the null-hypothesis, to check for other features in the spectrum which may have been concealed by that signal. This procedure is repeated un- 


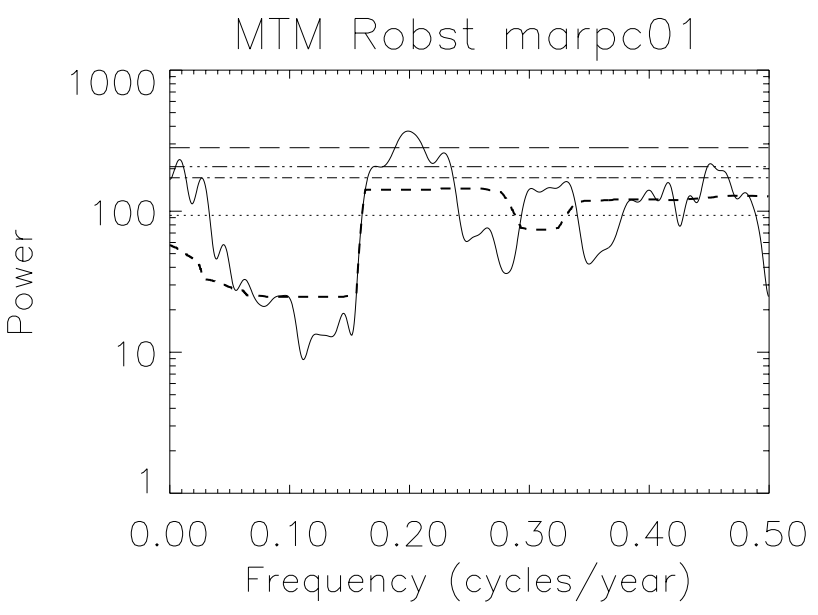

Fig. 2. Significant results of MTM for the pattern of March (ATL). The continuous line is the adaptive MTM spectrum; the various discontinuous lines are the median-smoothed spectrum (thick-dashed line), and the curves associated with the 50\%, 90\%, 95\%, and $99 \%$ confidence levels.

til a null-hypothesis that cannot be rejected is reached.

2. Filtering and reconstructing the time series. Another application of SSA is to filter the time series, reducing the background noise without losing any significant portion of the signal. Because the eigenvectors of the time series are not assumed to be sinusoidal, as in the case of the basis functions used in Fourier analysis methods, filtering with SSA is sometimes described as dataadaptive. Projecting the original time series onto the individual eigenvectors, one has the temporal principal components in each direction, which in SSA terms are called T-PCs. It is possible to reconstruct a filtered version of the time record based only on the significant TPCs. We take the set of dominant principal components and construct a filtered time series equal in length to the original series. The background noise is substantially reduced in this filtered time series. Then analysis by the Maximum Entropy Method (MEM), a high resolution spectral method, clearly reveals the frequency of the oscillations.

In sum, the procedure of the spectral analysis performed in this work will be first, to detect the significant oscillations by using the MCSSA test; second, to filter and to reconstruct the time series including only the detected signals; and finally, to analyze the reconstructed series using the Maximum Entropy Method (MEM).

\section{Results}

Due to the great number of time series (57), we shall explain in detail the procedure for one of them. The results for the complete set of series will be summarized in the corresponding tables. The example that we shall use to explain the procedure is the series corresponding to the Atlantic pattern of March. One of the reasons for using March ATL is that, at the end of the analysis, this case was found to be very interesting. The SSA-MTM toolkit (Dettinger et al., 1995) was used to carry out both the MTM and MCSSA spectral analyses.

\subsection{Multi-Taper Method}

Due to the shortness of our series (only 74 years), the MTM was performed using $p=2$ (i.e. a bandwidth of $4 / 74 \mathrm{cy}$ cles/year) and $K=3$. The robust red noise assumption was taken and the adaptive MTM spectrum was constructed. The spectrum was smoothed with a window of 0.15 cycles/year. Then a red noise model was fitted to the smoothed spectrum, obtaining the curves associated with the $90 \%, 95 \%$, and $99 \%$ confidence levels. The signals are then defined as the parts of the spectrum lying above the $99 \%$ curve.

\subsubsection{MTM results}

Figure 2 shows the MTM graph for the March ATL pattern, consisting of the power spectrum, the smoothed spectrum, and the confidence levels. The significant quasi-cycles are those whose power surpasses the $99 \%$ confidence level. The intervals of the significant quasi-cycles at the $99 \%$ confidence level are summarized in Table 2. Time series with no significant signals are represented by [-]. One notes that the ATL pattern presents significant periods in 4 of the 5 months in which it appears. Similarly, the SUR pattern appears in 10 months and has significant periods in 7 of them. By contrast, the CAN pattern is present during the whole year but has significant periods in only 2 months.

Most of the periods are between 2 and 6 years. Only two lie outside this interval ( 8 and 12 years). There are six months with one significant period: February (4 years), March (5 years), April (2.5 years), June (3.5 years), September (3.5 years), and October (3.5 years). There are two months without any significant period: July and August, the summer months. And there are four months with two significant periods: January ( 3 and 8 years), May (5 and 12 years), November (2 and 6 years), and December (2.5 and 6 years). The most frequent period is 3.5 years ( 7 events), followed by 2.5 years (6 events).

The MTM has thus reduced the initial set of 57 time series to a set of 27 series with significant oscillations. The following step is to study these 27 series in detail using MCSSA.

\subsection{MCSSA test: finding significant signals}

\subsubsection{March (ATL)}

1. One has first to apply data-adaptive MCSSA to find the data EOFs associated with the significant pure noise EOFs. Then, projecting both the data and 10000 surrogate series onto the data eigenbasis $E_{D}$ with a window width of $M=15$, and using 2.5 and 97.5 percentile limits, one obtains the data-adaptive eigenval- 
Table 2. MTM significant quasi-periods (year/cycle). Time series with no significant signal are represented by [-]

\begin{tabular}{|c|c|c|c|c|c|c|c|}
\hline Month & ATL & INT & SUR & POR & CAT & LEV & CAN \\
\hline JAN & $3.5-3.8$ & & {$[-]$} & & 8.3 & {$[-]$} & $3.1-3.2$ \\
\hline FEB & {$[-]$} & & $4.1-4.3$ & & $3.5-4.1$ & $3.7-4.0$ & {$[-]$} \\
\hline MAR & $4.7-5.2$ & & {$[-]$} & & {$[-]$} & {$[-]$} & {$[-]$} \\
\hline APR & & $2.3-2.4$ & $2.3-2.4$ & $2.3-2.6$ & 2.4 & {$[-]$} & {$[-]$} \\
\hline MAY & & {$[-]$} & $10.0-14.0$ & $4.7-5.2$ & {$[-]$} & [-] & [-] \\
\hline JUN & & & 3.7 & $3.4-3.5$ & & $3.4-4.0$ & 3.7 \\
\hline JUL & & & & {$[-]$} & & & {$[-]$} \\
\hline AUG & & & & {$[-]$} & & & {$[-]$} \\
\hline SEP & & {$[-]$} & $3.4-3.6$ & {$[-]$} & {$[-]$} & {$[-]$} & {$[-]$} \\
\hline OCT & & {$[-]$} & {$[-]$} & {$[-]$} & {$[-]$} & 3.4 & {$[-]$} \\
\hline NOV & $2.0-2.1$ & & $2.0-2.1$ & & $5.3 / 5.9$ & 5.8 & {$[-]$} \\
\hline DEC & $2.5 / 2.7$ & & $2.4-2.5 / 2.6-2-8$ & & $5.5-5.8 / 2.6-2.7$ & $5.5-6.2$ & {$[-]$} \\
\hline
\end{tabular}
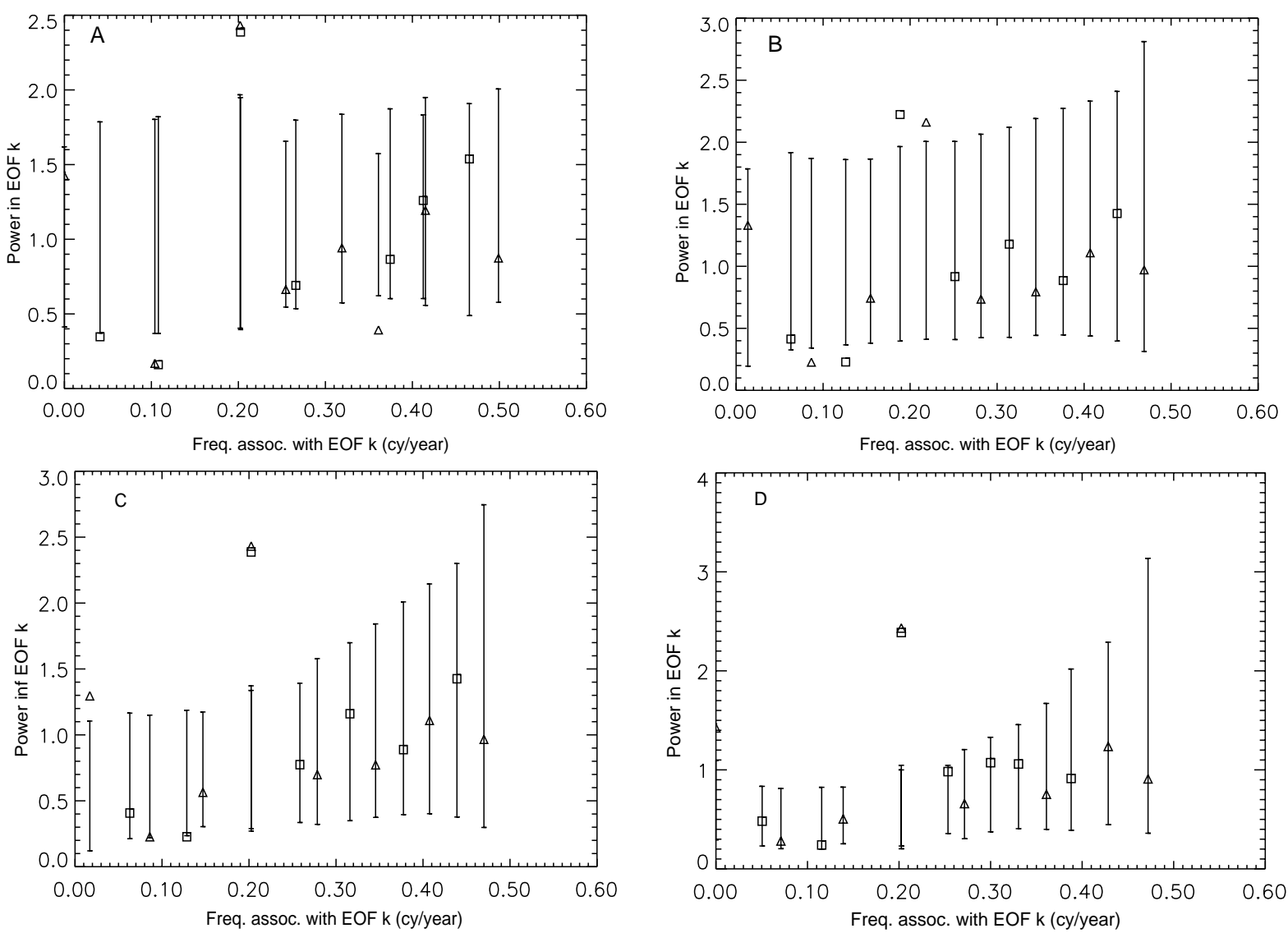

Fig. 3. Monte Carlo SSA of March (ATL): projection onto (a) the data-adaptive basis, (b) red noise null hypothesis basis, (c) composite null hypothesis basis (including data EOFs 1 and 2), (d) composite null hypothesis basis (including data EOFs 1, 2 and 3). In all cases the window length was $M=15 \mathrm{yr}$. The error bars denote 97.5 and 2.5 percentiles of a 10000 surrogate series.

ues and the surrogate data bars. These are plotted against the frequency associated with their corresponding EOFs. Since SSA EOFs are not pure sinusoidal functions, identifying a single frequency with each EOF is not straightforward. The association is made by maximizing the squared correlation with a sinusoidal func- tion. If the correlation is maximum with a sine function, the EOF is odd and the corresponding eigenvalue will be plotted with a square. If the correlation is maximum with a cosine function, the EOF is even and the eigenvalue is plotted with a triangle. A sine-cosine oscillatory pair thus appears when two eigenvalues (a square and a 
Table 3. Summary of MCSSA test results, with $M=15$

\begin{tabular}{cc}
\hline Pattern & Periods (years) \\
JAN(atl) & 3.7 \\
JAN(can) & 3.2 \\
FEB(sur) & 4.1 \\
FEB(lev) & 4.0 \\
FEB(cat) & 4.0 \\
MAR(atl) & $5.0 ;$ trend \\
MAY(sur) & 11.0 \\
JUN(lev) & 3.7 \\
JUN(por) & 3.5 \\
SEP(sur) & 3.4 \\
DEC(lev) & 5.8 \\
\hline
\end{tabular}

triangle) lie above their surrogate data bars. In Fig. 3a one sees that the first and second EOFs are significant and are located just over the 0.20 cycles/year frequency.

2. The MCSSA test of the pure noise null hypothesis (projecting both data and surrogates onto the $E_{N}$ noise basis) is performed with a window width of $M=15$ and 10000 Monte Carlo surrogate data series. The eigenvalues and the surrogate data bars (percentile limits: $2.5,97.5)$ are again plotted against the frequency associated with their corresponding EOFs (Fig. 3b). Note how the EOFs of the red noise covariance matrix are regularly separated by almost exactly $1 /(2 \mathrm{M})$. A sine-cosine oscillatory pair appears around the 0.20 cycles/year frequency. They are close together but not superimposed. This appearance of at least one significant eigenvalue is indicative of the presence of a quasi-oscillation different from noise.

Comparing the data-adaptive projection (Fig. 3a) with the pure noise projection (Fig. 3b) shows that the data EOFs 1 and 2 are associated with the pure noise EOFs 1 and 2.

3. We then assume that the data EOFs 1 and 2 are associated with the signal, and include them as signal in the composite null hypothesis. Projection onto the composite null hypothesis basis tends to pair up the signal EOFs just over the frequency corresponding to the quasi-oscillation, in this case 0.20 cycles/year (Fig. 3c). If oscillations other than those included in the null hypothesis existed, then new significant oscillatory pairs would appear. These pairs would have to be included in a new null hypothesis in the following iteration of the procedure, continuing the analysis until no new eigenvalue appears as significant. In the present case, there is one more significant eigenvalue. It corresponds to the 4th ranked of the data eigenbasis. Its secular period indicates a trend. We include this new significant EOF into another composite null hypothesis including the 1st, 2nd and 4th data EOFs as signals (Fig. 3d). Now, no further significant eigenvalues have appeared,
Table 4. Analysis using different window widths. $M=$ Window width (years), Variance: percentage of the reconstructed component with respect to the original series

\begin{tabular}{cccccc}
\hline & $M=12$ & $M=15$ & $M=18$ & Stability & Variance (\%) \\
Pattern & Prd (yr) & Prd (yr) & Prd (yr) & & M=15 \\
JAN(atl) & - & 3.7 & 3.7 & NS & 12.9 \\
JAN(can) & 3.2 & 3.2 & 3.2 & S & 12.7 \\
FEB(sur) & - & 4.1 & - & NS & 13.4 \\
FEB(lev) & - & 4.0 & 4.0 & NS & 14.7 \\
FEB(cat) & 4.0 & 4.0 & 4.0 & S & 14.3 \\
MAR(atl) & $5.0 ;$ trend & $5.0 ;$ trend & $5.0 ;$ trend & S & 27.3 \\
MAY(sur) & - & 11.0 & - & NS & 13.4 \\
JUN(lev) & 3.7 & 3.7 & 3.7 & S & 33.3 \\
JUN(por) & - & 3.5 & 3.5 & NS & 13.8 \\
SEP(sur) & - & 3.4 & 3.4 & NS & 13.9 \\
DEC(lev) & - & 5.8 & 5.8 & NS & 14.3 \\
\hline
\end{tabular}

which means that the signal is a quasi-cycle of 5.0 years and a secular trend.

\subsubsection{Summary of the results}

The results of the applying the preceding process (with $M=$ 15 ) to each time series are summarized in Table 3 . Only these eleven patterns presented significant signals.

It is now necessary to check whether these significant quasi-oscillations are stable against changes in the window width. Some sensitivity of results to window width is inevitable due to the constraint that the EOFs must be orthogonal, but if a pair only appears for certain values of $M$, this is a reason to doubt its significance. But unfortunately, the converse is not true: the stability of an oscillatory pair does not assure its physical significance (Allen and Smith, 1996).

The entire process (pure noise projection and composite null hypothesis test) was repeated with $M=12$ and $M=18$, and some interesting results were found (Table 4) which will be discussed in the following subsection.

\subsubsection{The selected quasi-cycles: moving window analysis}

The analysis of signal stability by changing the window width revealed that not all the pre-selected signals are stable. Table 4 lists the results for the three windows, and shows how there are signals that are significant only for certain windows, whereas there are other signals that are significant for all three windows. The table gives the most important characteristics of the signals: their stability, the quasi-period, and the variance explained (in the case of $M=15$ ). This variance was computed as the sum of the variances explained by each of the significant EOFs associated with the quasi-cycle.

The signals can thus be classified into "stable" $(S)$, indicating the presence of eigenvalues in the three windows, and "non-stable" $(N S)$, indicating the absence of significant eigenvalues in some window. From Table 4, one ob- 
serves four stable quasi-oscillations (January (CAN), February (CAT), March (ATL), and June (LEV)). Also, the oscillations corresponding to March (ATL) and June (LEV) are well defined (in the sense that they lie clearly above the surrogate bars) at $M=15$ and $M=18$. The rest of the quasioscillations seem to be unstable and poorly defined. For that reason, the initial selection of significant quasi-cycles has to be questioned. Observing Table 4, one might conclude that the "best" quasi-cycle is the 3.7 years of June (LEV), because it is stable, well defined (at $M=15$ and $M=18$ ), and explains $33.3 \%$ of the variance, better than, for example, March (ATL) which, while it is stable, and well defined (at $M=15$ and $M=18$ ), only explains $27.3 \%$ of the variance. That conclusion could be misleading.

Therefore, a moving window analysis is performed in order to assess the dates for which the oscillations remain significant. The moving window analysis slides a window of length $S$ down a series of length $N$, generating a set of $N-S+1$ sub-series that will be studied, one by one, with MCSSA. If the oscillation of the original series remains significant in each of the sub-series, one can conclude that the oscillation is stable over the entire original period $(N)$, i.e. the oscillation is not a particular event during a certain temporal interval that appears as significant in the analysis of the whole period. To this goal, MCSSA was performed with $M=12$ instead of $M=15$, since with a moving window width of $S=61$, a lower value of $M$ will preserve the significance level. The window width $S=61$ generates 14 subseries from each series. These were analysed using MCSSA under the null hypothesis of pure noise.

The results showed that the oscillation of June (LEV) was significant only around the first part of the 1919-1992 period and was not significant in the 1927-1992 period. It seems that the oscillation was so strong in this first part of the time series that it remained significant in the analysis of the whole period. Only by analyzing the sub-series can one delimit the period of existence of the oscillations. In our case, the oscillations of January (CAN), February (CAT), and March (ATL) were significant during the whole 1919-1992 period. In all three cases, the significance of the oscillations tended to decrease in the last years of the period.

For the case of March (ATL), there was an interesting evolution of the ranking of the eigenvalues in the different subseries. The two eigenvalues (ranked 1 and 2) associated with the quasi-cycle of 5 years remain in this position in all the sub-series. The third position, however, is occupied by an eigenvalue corresponding to a quasi-cycle of 2.5 years and not by the eigenvalue corresponding to the trend, which is situated in the last place of the ranking from sub-series 1 to sub-series 8 . In sub-series 9 , the eigenvalue corresponding to the secular trend begins to climb in rank, arriving at the third position by sub-series 14 . This concurs with the form of the trend (Fig. 4) obtained by reconstructing the series including eigenvalue 3 of the pure noise MCSSA with $M=15$ (whole period). One observes that the downward trend starts around the 1960s and continues until the 1990s (the last three years of the series), when it seems to bottom out.
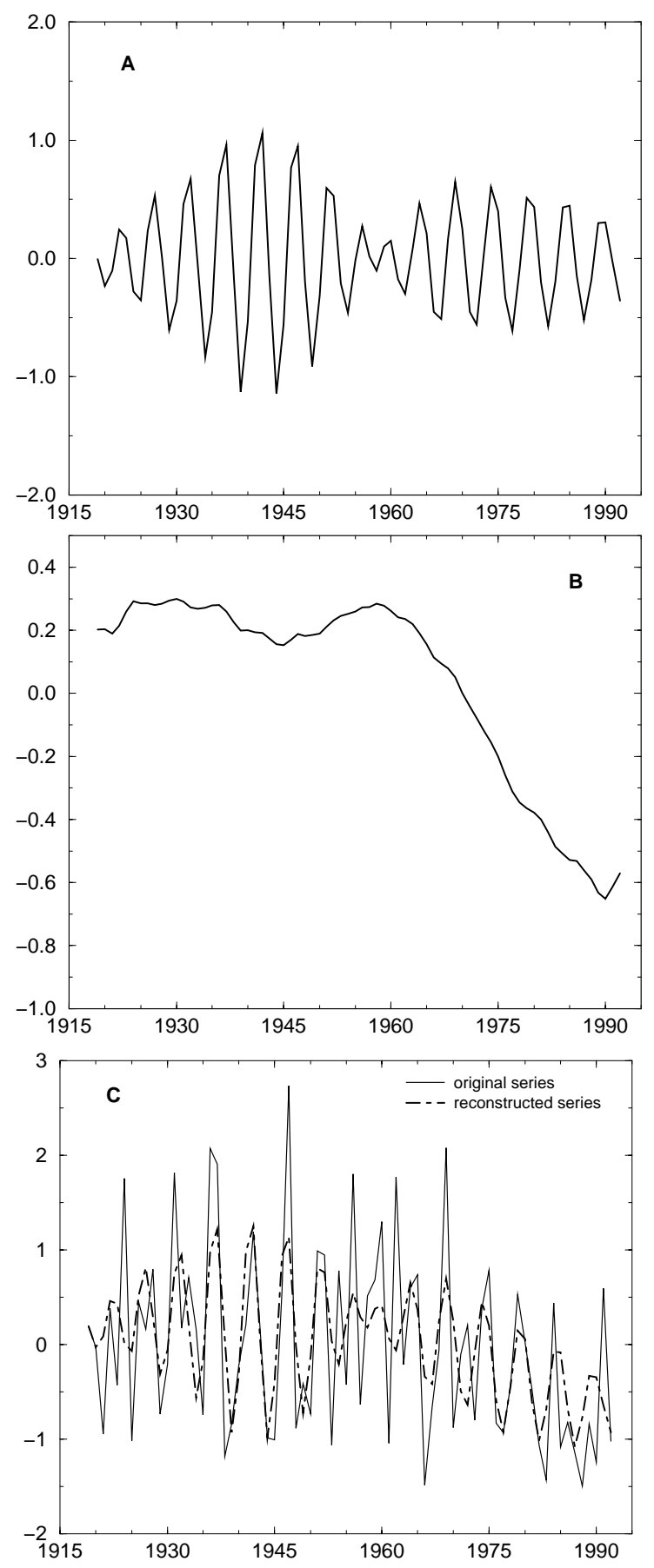

Fig. 4. Reconstructed series for March (ATL): (a) including only EOFs 1 and 2, corresponding to the quasi-oscillation, (b) including only EOF 3, corresponding to a long-term trend, (c) original time series plus reconstructed signal including EOFs 1, 2, and 3.

The case of January (ATL) is particular and calls for attention. When the pure noise MCSSA was performed with $M=12$, no significant oscillations were found at the $95 \%$ level of confidence. But in the moving window analysis of this series with $M=12$ and $95 \%$, there appears a significant eigenvalue in 9 of the sub-series. This result suggested the 

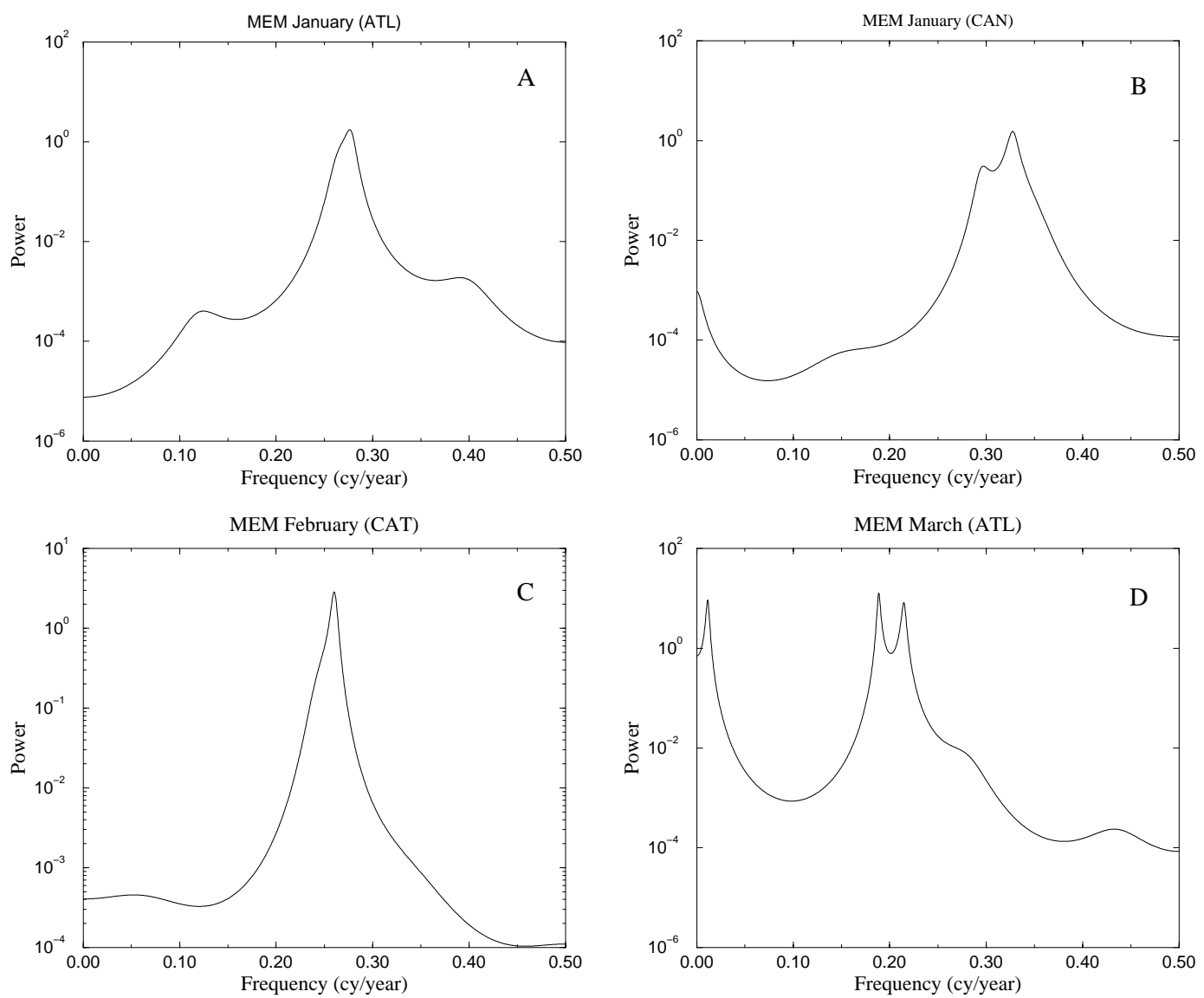

Fig. 5. Maximum Entropy Method (MEM) spectrum of the reconstructed series: (a) MEM spectrum of the reconstructed series of January (ATL) including only EOF 1, corresponding to a 3.7 year oscillation. (b) MEM spectrum of the reconstructed series of January (CAN) including only EOF 1, corresponding to a 3.4-3.1 year quasi-oscillation. (c) MEM spectrum of the reconstructed series of February (CAT) including EOF 1, corresponding to a 4.0 year oscillation. (d) MEM spectrum of the reconstructed series of March (ATL) including EOFs 1, 2, and 3, corresponding to a long-term trend, and a 5.2-4.7 year quasi-oscillation.

repetition of the analysis at a $90 \%$ confidence level. Now, a significant eigenvalue was found for the three windows and in the composite null hypothesis analysis. The oscillation was also significant in the whole set of sub-series of the moving window analysis. For these reasons, this quasi-cycle of 3.7 years of January (ATL) merits inclusion among the selected quasi-oscillations.

\subsection{Studying the significant signals}

Once significant stable signals were found, we could make a detailed study of their associated frequencies. We obtained the filtered time series by reconstruction with the significant data EOFs. Figure 4 shows the reconstructed quasioscillations and the original time series plus the filtered signals for the March (ATL) pattern. These filtered signals were analyzed using the Maximum Entropy Method (MEM). Figure 5 shows the different signals. In Fig. 5a, one can observe a clear oscillation of 3.7 years in January (ATL). Figure $5 b$ shows the quasi-oscillation of 3.4-3.1 years (maximum at 3.1 years) present in January (CAN). Figure $5 \mathrm{c}$ is the oscillation of 4.0 years of February (CAT). Figure 5d, corresponding to March (ATL), shows the presence of a trend and a quasioscillation of 5.2-4.7 years.

\section{Composite maps}

Once the oscillations have been identified (quasi-cycles and trends), a composite analysis is performed, in order to detect any sea level pressure structure that occurs simultaneously at high values of the quasi-oscillations found for a certain precipitation regime. This could be a sign of the existence of possible relationships between these oscillations and sealevel pressure (SLP). We applied a composite analysis to the SLP anomalies and tested the average values for a significant difference from the mean using Student's t-test.

The series with the four best quasi-oscillations were selected as index series. The series were reconstructed by including the EOFs associated with the pure noise significant eigenvalues (i.e. including only the detected quasi-cycles and trends). The composite analysis was performed with:

1. The reconstructed series of January (ATL), including EOF 1 corresponding to the quasi-cycle of 3.7 years; 

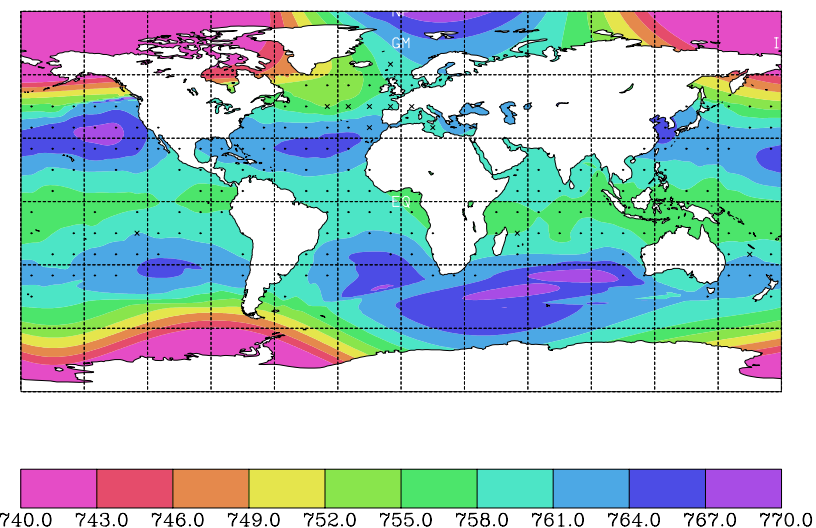

Fig. 6. Composite map of SLP (in $\mathrm{mm} \mathrm{Hg}$ ) for March corresponding to wet years according to the pattern March (ATL). Dots correspond to $10^{\circ} \times 10^{\circ}$ boxes where Student's t-test for the difference in the means of wet and non-wet years was not significant at a confidence level of $99 \%$. Crosses correspond to boxes where that difference was significant.
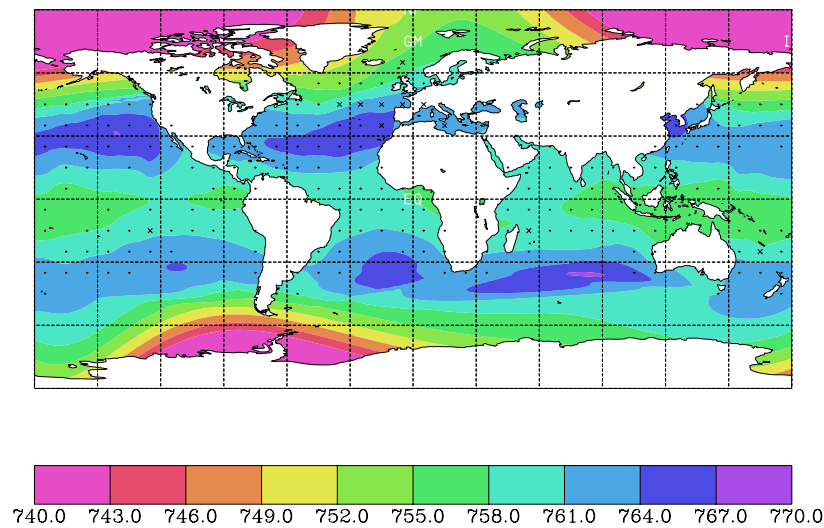

Fig. 7. Composite map of SLP for March corresponding to non-wet years according to the pattern March (ATL).

2. The reconstructed series of January (CAN), including EOF 1 corresponding to the quasi-cycle of 3.2 years;

3. The reconstructed series of February (CAT), including EOF 1 corresponding to the quasi-cycle of 4.0 years;

4. The reconstructed series of March (ATL), including EOFs 1, 2, and 4 corresponding to the quasi-cycle of 5.0 years and the secular trend.

These index series were composited with the sea-level pressure series, obtained from COADS (Comprenhensive Ocean-Atmosphere Data Set, (Woodruff et al., 1987)), for the period 1919-1992 and using boxes of $10^{\circ}$ latitude $\times$ $10^{\circ}$ longitude. A threshold of $0.6 \sigma$ separated the wet years from the non-wet years. Using Student's t-test, with a confidence level of $95 \%$, we found that only two reconstructed series showed significant differences between the means of SLP corresponding to wet years and non-wet years: January (ATL) and March (ATL). The composite maps of these series
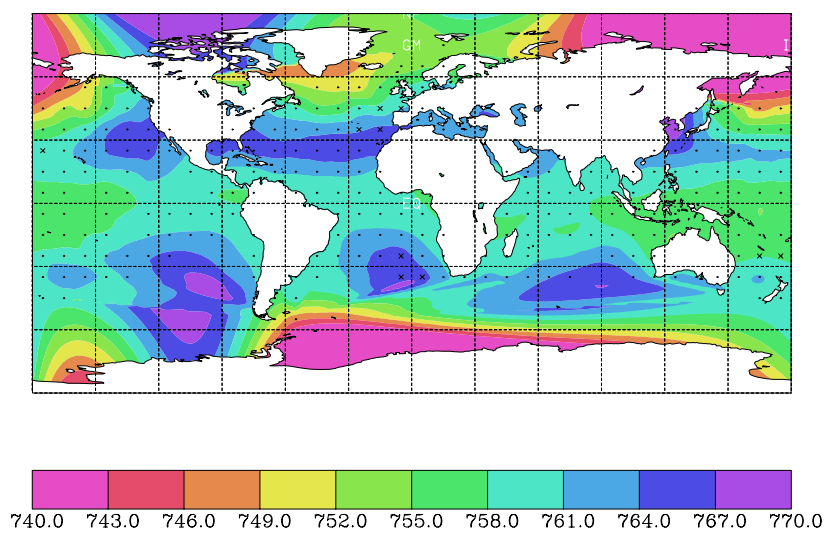

Fig. 8. Composite map of SLP for January corresponding to wet years according to the pattern January (ATL).
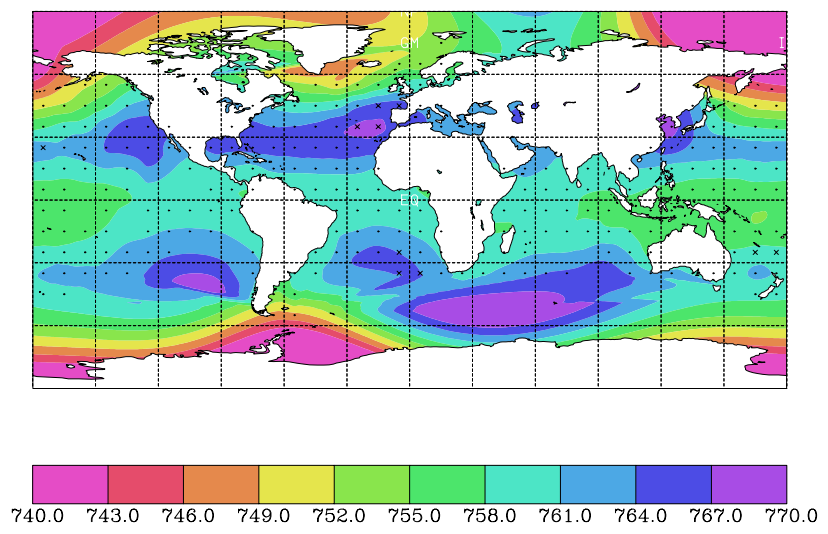

Fig. 9. Composite map of SLP for January corresponding to nonwet years according to the pattern January (ATL).

are shown in Figs. 6-9, in which the dots indicate the boxes with no significant values under the t-test and the crosses indicate the significant boxes. Less significant but still very interesting are the structures observed in the composite maps corresponding to the reconstructed series of January (CAN). No other significant results were found in the rest of the composite maps.

Figures 6 and 7 show the composite maps corresponding to the wet and non-wet years of the reconstructed series of March (ATL). There are four significant boxes, in the area of the North Atlantic Ocean and in the Cantabrian zone. Figures 8 and 9 represent the maps for the wet and non-wet years of the reconstructed series of January (ATL). There are four significant boxes in the Atlantic zone near the Peninsula. The location of the high pressure structures is different during the wet years and during the non-wet years of the same series. There is also a similarity between the structures of the wet years of January (ATL) and of March (ATL), and also of the non-wet years of the same two series.

The two different circulation patterns for wet and non-wet years for the ATL pattern (Figs. 6 and 7 for the case of March, and Figs. 8 and 9 for the case of January) seem to be a mani- 

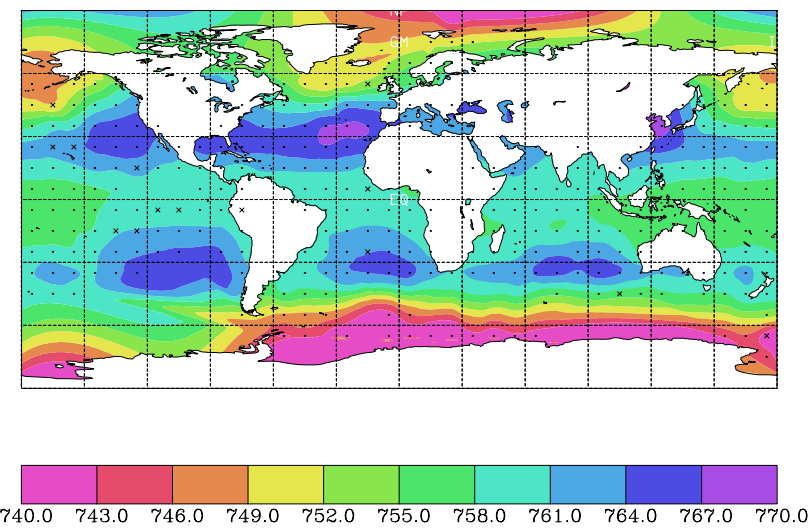

Fig. 10. Composite map of SLP for January corresponding to wet years according to the pattern January (CAN).
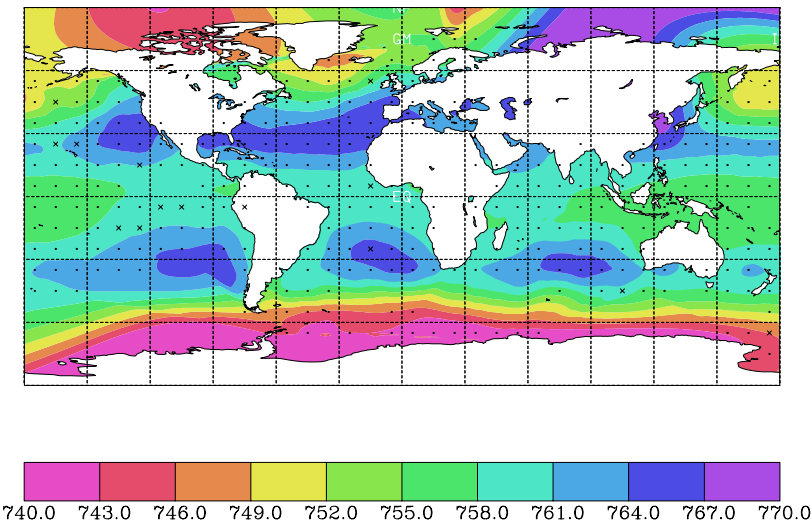

Fig. 11. Composite map of SLP for January corresponding to nonwet years according to the pattern January (CAN).

festation of the North Atlantic Oscillation (NAO).

The composite maps for the wet years for the ATL pattern (Figs. 6 and 8) show a zonal mean circulation over all the Atlantic Coast of Europe including the Iberian Peninsula, with only the low latitudes being affected by the semipermanent high pressure systems. This situation corresponds to a low NAO index. This atmospheric situation allows cyclonic perturbations to cross the Peninsula, leading to large amounts of rainfall over Portugal and most of Spain.

These results for January and March agree with the work of Zorita et al. (1992), who found the NAO to be one of the major factors of the winter precipitation over the Iberian Peninsula.

On the contrary, the composite maps for the non-wet years for the ATL pattern (Figs. 7 and 9) show that the high pressure belt has expanded northwards to cover the Iberian Peninsula. This situation corresponds to a high NAO index resulting from the very high values of the pressure over the Azores. Now, the zonal circulation has moved to higher latitudes with the flows over the Iberian Peninsula being weaker and with a highly meridional character. This keeps the tracks of low pressure systems to the north, far from the Iberian Peninsula,
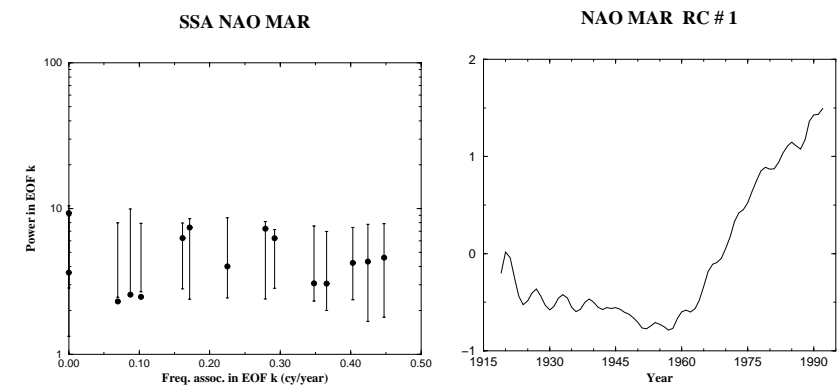

Fig. 12. Singular Spectrum Analysis for NAO March (left) and reconstructed component with EOF 1 (right).

resulting in very dry periods.

The contrary situation occurs for the CAN precipitation regime in January. The wet years (Fig. 10) occur for a high NAO index with a high pressure structure located over the Azores. Under these conditions, the Atlantic air masses cross the Iberian Peninsula from northwest to southeast, leading to precipitation at the northern part of the Cantabric mountain chain, which is the region associated with the CAN pattern. The non-wet years (Fig. 11) correspond to conditions of a low NAO index. In this situation, the Atlantic air masses cross the Iberian Peninsula from southwest to northeast, arriving dry and warm at the Cantabrian coast after suffering the Foehn effect over the Cantabrian mountain chain.

It is surprising the decrease in rainfall found for the low frequency component in the ATL pattern in March (Fig. 4). No similar situation is found in any other pattern or month. This decrease in March precipitation over the Iberian Peninsula has been reported in other works for Portugal (CorteReal et al., 1998; Trigo and DaCamara, 2000) and for the Iberian Peninsula (Serrano et al., 1999b). In addition, an important increase in the monthly precipitation for the months of March (and October to a lesser extent) on the west coast of Ireland has been reported by Kiely et al. (1998).

In order to look for the causes of this trend, taking into account the suggested relationship between the NAO and the monthly precipitation characterized by the ATL regime, an SSA for the NAO index (based on the difference of normalized sea level pressure (SLP) between Ponta Delgada, Azores and Stykkisholmur/Reykjavik, Iceland, see http://www.cgd. ucar.edu/ $\sim$ jhurrell/nao.html\#oseas) for March has been performed. Figure 12 shows the spectrum of eigenvalues versus frequency. It can be seen that the leading eigenvalue is associated with a trend. The series reconstructed with this eigenvalue is shown in Fig. 12. One observes a significant increase in the NAO index from the beginning of the sixties. This behavior agrees with the decrease shown by the precipitation described by the ATL pattern during March (Fig. 4).

Further SSA analyses were performed on the NAO index for the other winter months (December, January and February) and no similar trend was found for any of them, thus, confirming that the trend occurs only in March. 


\section{Discussion of the results}

The use of two quite different methods of spectral analysis allows one to glean more information from the time series. The periods or quasi-periods selected as significant in this work were those that satisfy the requirements for a signal in both methods.

The MTM offered a preliminary view of the characteristics of the series, and focused our attention on a group of 27 series and 31 quasi-oscillations. The MTM results were given in Table 2. These quasi-periods are temporal intervals defined by the sections of the spectral peaks that surpass the $99 \%$ confidence level. There was a notable absence of patterns and quasi-periods during the summer months (July and August) consistent with the very occasional nature of precipitation in this season. Most of the quasi-periods ranged from 2 to 6 years. Only two of them lay outside this interval: the signal of the CAT pattern of January (8 years) and that of the SUR pattern of May (10-14 years). It has to be kept in mind that since the series are 74 years long, periods longer than 8-10 years have less statistical significance than those of 3-5 years.

The MCSSA results (Table 3) selected only 11 quasioscillations, with more precise frequencies, that were within the temporal intervals defined by the MTM. The analysis of the stability of the signals (Table 4) indicated that there are only four stable quasi-cycles (significant eigenvalues for the three windows, 12,15 and 18 years) at the $95 \%$ confidence level:

1. The quasi-oscillation of 3.2 years in the Cantabrian pattern of January;

2. The quasi-oscillation of 4.0 years in the Catalonian pattern of February;

3. The quasi-oscillation of 5.0 years and a trend in the Atlantic pattern of March;

4. The quasi-oscillation of 3.7 years in the Levante pattern of June;

and one stable quasi-cycle at the $90 \%$ confidence level: the quasi-oscillation of 3.7 years in the Atlantic pattern of January.

The moving window analysis of the five stable quasioscillations showed the oscillations of January (CAN), January (ATL), February (CAT), and March (ATL) to be defined over the whole study period 1919-1992. The quasioscillation of June (LEV) appeared to be associated with only the first part of the period (1919-1927): in the rest of the period, the oscillation was not significant. Therefore, we rejected the oscillation of June.

The reconstruction of the filtered signals (Fig. 4) showed the March (ATL) trend to be clearly downward which could be justified by the increase in the NAO index for that month. The MEM analysis of the reconstructed signals (Fig. 5) showed that the 5.0 year oscillation of March (ATL) appeared inside a quasi-oscillation of 5.2-4.7 years. The 3.2 year oscillation of January (CAN) appeared inside a quasioscillation of 3.4-3.1 years.

The study of the composite maps of the selected quasioscillations indicated that:

1. The high values of precipitation generated by the quasicycle of the ATL pattern in March exist simultaneously with a high pressure structure over the North Atlantic Ocean;

2. The high values of precipitation generated by the quasicycle of the ATL pattern in January exist simultaneously with that same pressure structure over the North Atlantic Ocean;

3. The high values of precipitation generated by the quasicycles of the CAN pattern in January exist simultaneously with another pressure structure over the Galicia zone and Cantabrian Sea.

Among the significant quasi-oscillations found in this work, the downward trend in March has been reported before for Portugal (Corte-Real et al., 1998; Trigo and DaCamara, 2000) and for the Iberian Peninsula (Serrano et al., 1999b). With reference to the rest of the significant oscillations, which correspond to short periods between 3 and 5 years, similar results have been reported by RodriguezPuebla et al. (1998) using annual time series, and by García et al. (2002) using continuous quarterly data.

As was established before in the composite analysis, sea level pressure patterns which occur simultaneously with the oscillations seem to be related to the NAO. A spectral analysis of the NAO index (Hurrel and Van Loon, 1997; Wunsch, 1998; Robertson, 2001) reveals a quasi-oscillation with a period of about 2.5 years which is somewhat shorter than ours. However, Tourre et al. (1999), studying spatiotemporal patterns of joint sea surface temperature and sea level pressure variability in the Atlantic Ocean, found a 3.5 year period which corresponds to an SLP dipole-like pattern similar to the NAO, and a 4.4 year period related to pressure anomalies located between Iceland and Greenland. Both patterns could affect the strength of westerly winds, and therefore the precipitation regimes, over a large area including the Iberian Peninsula. Also, Venegas and Mysak (2000) found periods around 5 years, 2.7 years and 2.1 years for North Atlantic SLP anomalies.

It is cautiously concluded that the short periods detected in the analysis could be related to the oscillations found in the sea level pressure over the North Atlantic Ocean.

Acknowledgements. This work was supported by the Spanish CICYT under Project CLI99-0845-C03-03. Also thanks are due to the SSA-MTM group for providing us with the SSA-MTM toolkit and to the Spanish Instituto Nacional de Meteorología for providing us with the rainfall series.

Topical Editor J.-P. Duvel thanks two referees for their help in evaluating this paper. 


\section{References}

Allen, M. R. and Smith, L. A.: Monte Carlos SSA: Detecting irregular oscillations in the presence of colored noise, J. Climate, 6 , 3373-3402, 1996.

Berger, A. L., Mélice, J. L., and Hinnov, L.: A strategy for frequency spectra of Quaternary climate records, Climate Dynamics, 5, 227-240, 1991.

Broomhead, D. S. and King, G.: On the Qualitative Analysis of Experimental Dynamical Systems, Adam Hilger, Bristol, 1986.

Corte-Real, J., Qian, B., and Xu, H.: Regional climate change in portugal: Precipitation varibility associated with large-scale atmospheric circulation, Int. J. Climatol., 18, 619-635, 1998.

Dettinger, M., Ghil, M., Strong, C. M., Weibel, C. M., and Yiou, P.: Software spedites singular-spectrum analysis of noisy time series, Eos, Trans. American Geophysical Union, 76, 12, 14-21, 1995.

Elsner, J. and Tsonis, A.: Singular Spectrum Analysis: A New Tool in Time Series Analysis, Plenum Press, 1996.

Fraedrich, K.: Estimating the dimensions of weather and climate attractors, Atmospheric Science, 43, 419-432, 1986.

Fraedrich, K. and Ziehmann-Schlumbohm, C.: Predictability experiments with persistence forecast in red-noise atmosphere, Quart. J. Roy. Meteor. Soc., 120, 387-428, 1994.

García, J. A., Serrano, A., and Gallego, M. C.: A spectral analysis of iberian peninsula monthly rainfall, Theor. Appl. Climatol., 71, 77-95, 2002.

Ghil, M. and Vautard, R.: Interdecadal oscillations and the warm trend in global temperature time series, Nature, 350, 324-327, 1991.

Gilman, D. L., Fuglister, F. L., and Mitchell, J. M. J.: On the power spectrum of red noise, J. Atmos. Sci., 20, 182-184, 1963.

Hurrel, J. and Van Loon, H.: Decadal variations in climate associated with the north atalntic oscillation, Climatic Change, 36, 301-326, 1997.

Jollife, I.: Principal Component Analysis, Springer-Verlag, 1986.

Kiely, G., Albertson, J. D., and Paralange, M. B.: Recent trends in diurnal variation of precipitation at valentia on the west coast of ireland, J. Hidrol., 208, 1998.

Kuo, C., Lindberg, C. R., and Thomson, D. J.: Coherence established between atmospheric carbon dioxide and global temperature, Nature, 343, 709-714, 1990.

Lanzerotti, L., Thomson, D. J., Maclennan, C. G., and Medford, L. V.: Electromagnetic study of the Atlantic continental margin using a section of a trasatlantic cable, J. Geophys. Res., 91, 7417-7427, 1986.

Lindberg, C. R.: Multi-Taper Spectral Analysis of Terrestrial Free Oscillations, Ph.D. thesis, Scripps Institution of Oceanography, University of California, San Diego, 1986.

Mann, M. E. and Lees, J. L.: Robust estimation of background noise and signal detection in climatic time series, Clim. Change, 33, 409-445, 1996.

Mann, M. E., Park, J., and Bradley, R. S.: Global interdecadal and century-scale climate oscillations during the past five centuries,
Nature, 378, 266-270, 1995.

Park, J., Lindberg, C. R., and Vernon III, F. L.; Multi-Taper spectral analysis of high-frequency seismograms, J. Geophys. Res., 92, 12 675-12 684, 1987.

Percival, D. B. and Walden, A. T.; Spectral Analysis for Physical Applications, Cambridge University Press, Cambridge, UK., 1986.

Robertson, A.; Influence of the ocean-atmosphere interaction on the artic oscillation in two general circulation models, J. of Climate, 14, 3240-3254, 2001.

Rodriguez-Puebla, C., Encinas, A. H., Nieto, S., and Garmendia, J.: Spatial and temporal patterns of annual precipitation variability over the iberian peninsula, Int. J. Climatol., 18, 299-316, 1998.

Serrano, A., García, J. A., Mateos, V. L., Cancillo, M. L., and Garrido, J.: Monthly Modes of Variation of Precipitation over the Iberian Peninsula, J. Climate, 12, 2894-2919, 1999a.

Serrano, A., Mateos, V. L., and García, J. A.: Trend Analysis of Monthly Precipitation over the Iberian Peninsula for the period 1921-1995, Phys. Chem. Earth (B), 24, 85-90, 1999b.

Slepian, D.: Prolate spheroidal wave functions, Fourier analysis and uncertainty, V. The discrete case, Bell Syst. Tech. J., 57, 13711429, 1978.

Thomson, D.: Quadratic-inverse spectrum estimates: applications to paleoclimatology, Phil. Trans. R. Soc. London A, 332, 539597, 1990a.

Thomson, D.: Time series analysis of Holocene climate data, Phil. Trans. R. Soc. London A, 330, 601-616, 1990 b.

Thomson, D. J.: Spectrum estimation and harmonic analysis, Proc. IEEE, 70, 1055-1096, 1982.

Tourre, Y. M., Rajagopalan, B., and Kushnir, Y.: Dominant patterns of climate variability in the atlantic ocean during the las 136 years, J. of Climate, 12, 2285-2299, 1999.

Trigo, R. M. and DaCamara, C. C.: Circulation weather types and their influence on the precipitation regime in portugal, Int. J. Climatol., 20, 1559-1581, 2000.

Vautard, R. and Ghil, M.: Singular Spectrum Analysis in nonlinear dynamics, with applications to paleoclimatic time series, Physica D, 35, 395-424, 1989.

Venegas, S. A. and Mysak, L. A.: Is there a dominant timescale of natural climate variability in the arctic, J. Climate, 13, 34123434, 2000.

Woodruff, S. D., Slutz, R. J., Jenne, R. L., and Steurer, P. M.: A comprehensive ocean-atmosphere data set., Bull. Amer. Meteor. Soc., 68, 1239-1250, 1987.

Wunsch, C.: The interpretation of the short records, with comments on the north atlantic and southern oscillations, Bulletion of the AMS, 80, 245-255, 1998.

Yiou, P., Jouzel, J., Johnsen, S., and Rognvaldsson, O. E.: Rapid oscillations in Vostok and GRIP ice cores, Geophys. Res. Lett., 22, 2179-2182, 1995.

Zorita, E., Kharin, V., and Von Storch, H.: The atmospheric circulation and sea surface temperature in the north atlantic area in winter: Their interaction and relevance for Iberian precipitation, J. Climate, 5, 1097-1108, 1992. 